\title{
Consciousness, EEG and depth of anaesthesia monitoring
}

\author{
Peng Wen
}

Published online: 18 December 2012

(C) Australasian College of Physical Scientists and Engineers in Medicine 2012

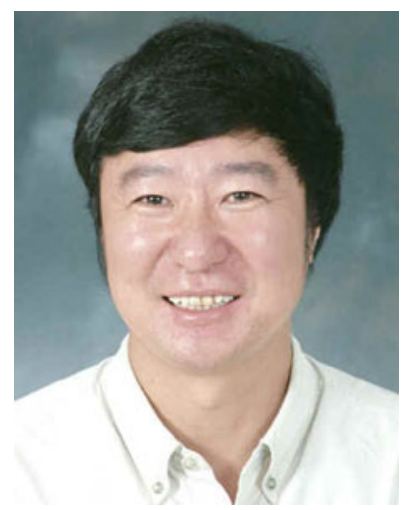

Dr Peng (Paul) Wen, APESM Associate Editor

\section{Introduction}

Many surgical procedures would not be possible without patient entering a state of unconsciousness. The essential features of a successful general anaesthesia, displayed by the patient, are a reversible loss of consciousness (LOC) with a lack of movement, a lack of awareness, unresponsiveness to painful stimuli and a lack of recall of the surgical intervention. Inadequate general anaesthesia may lead to intraoperative awareness with recall or to prolonged recovery and an increased risk of postoperative complications for the patient. An important contributing factor to inadequate general anaesthesia is our current limited ability to assess the levels of consciousness.

P. Wen $(\bowtie)$

Faculty of Engineering and Surveying, University of Southern

Queensland, Toowoomba, QLD 4350, Australia

e-mail: pengwen@usq.edu.au
Adequate surgical anaesthesia must achieve three goals: immobility, amnesia, and absence of awareness. It was widely assumed that all these actions were accomplished at some unitary site, after the evidence of anaesthetic lipophilicity was presented by Meyer and Overton [1]. A body of evidence has now accumulated demonstrating that for many anaesthetic agents, the dose required to suppress consciousness exceeds the amnestic dose but is substantially less than that required for surgical immobility during noxious stimuli [1]. This suggests that these three dimensions may be mediated by different regions of the central nervous system. As pointed out by Rampil [2], the variability among anaesthetics of the ratios of concentrations needed to suppress consciousness, to block memory, and to achieve surgical immobility further invalidate the unitary hypothesis. A comprehensive explanation of the mechanism by which anaesthetics cause LOC has not yet been developed. Campagna et al. [3] have recently provided a review of current understanding of the molecular mechanisms of anaesthesia, summarizing evidence showing that inhaled anaesthetics achieve immobilization by depressing the spinal cord, whereas amnesic actions are mediated within the brain. Their documents indicate that subtle differences in the clinical actions of inhaled anaesthetics may be attributed to distinct actions on a number of critical molecular targets. Although this evidence makes it clear that neuronal actions and interactions at many different levels and in many different brain tissues are altered or disrupted by anaesthetic drugs, it does not explain why these different more or less discrete effects have the common global effect of causing LOC.

The search for a reliable depth of anaesthesia monitor, that would enable the objective, reproducible and continuous measurement of anaesthetic depth, has led to the development of electroencephalogram (EEG) or acoustic evoked 
potentials (AEP) based monitors $[4,5]$. These enable continuous monitoring even during the period a patient has lost all responses to external stimuli. The process of monitoring depth of anaesthesia and administration of a general anaesthetic during surgery is a closed-loop control system where the human is responsible for reasoning and action. Anaesthetists play the roles of controller and actuator by deciding on the amount of anaesthetic and when to administer it at what rates. On the other hand, the activity of monitoring is performed automatically by commercially available depth of anaesthesia monitors. Together they form a closed-loop control system. The proposed control systems are most often built around a well established DoA monitor, which is now standard equipment for anaesthesia monitoring. However, these DoA monitors have undergone rigorous testing, which have shown drawbacks. Therefore the problem of constructing an ideal DoA monitor is still unsolved [6-9]. We currently rely on indirect measurable quantities, which give us a relatively reliable measure of the state of awareness. Several algorithms and methods have been developed for assessment of the DoA in the last decade. Their common denominator is the reduction of information, which comes from one or more complex signals, into a single feature, which reflects the state of consciousness [8-12].

A number of EEG based technologies are available. The first commercially successful product for routine intra-operative EEG monitoring of anaesthetic depth was produced by Aspect Medical Systems (Newton, Massachusetts) and given the name BIS (derived from bispectral analysis, which forms part of the algorithm used to interpret the EEG). Other products for monitoring anaesthetic depth include the Patient State Analyser (PSA) 4000 from Physiometrix; the SEDLine monitor from Hospira, Inc.; the A-Line AEP Monitor/2 by Danmeter; the Entropy monitor by Datex-Ohmeda; the hand-held Snap monitor from Everest Biomedical Instruments; and the Narcotrend and Cerebral State Monitor from Danmeter of Denmark. Amongst these products, BIS is the most thoroughly studied and most widely used. Perhaps inappropriately, BIS is seen as a "gold standard" for the evaluation and comparison of new products. However, it has been identified that [7]:

- The currently available monitoring algorithms do not account for all anaesthetic drugs, including ketamine, nitrous oxide and halothane.

- Electromyography (EMG) and other high-frequency electrical artifacts are common and interfere with EEG interpretation.

- Data processing time produces a lag in the computation of the depth-of-anaesthesia monitoring index.

- The EEG effects of anaesthetic drugs are not good predictors of movement in response to surgical stimulus because the main site of action for anaesthetic drugs to prevent movement is the spinal cord.

- The use of depth of anaesthesia monitoring in children is not as well understood as in adults.

The difficulty in tackling depth-of-anaesthesia is for anaesthetists to determine what constitutes 'adequate' anaesthesia for an individual patient. Clinical parameters, such as heart rate and blood pressure, have given results that are too inconsistent to be useful in this respect and the precise concentration of anaesthetic agents required to guarantee lack of recall in an individual remains unknown. It is this difficulty, coupled with the fear of eliciting awareness and all its long-term consequences, that has driven the search for a more reliable and more accurate 'depth-of-anaesthesia' monitor. Currently the vast majority of patients tend to receive more than enough anaesthetic when anaesthesia is administered without the guidance of depth of anaesthesia monitoring.

Anaesthetists now use a variety of different indicators to monitor DoA, many of which rely upon monitoring more accurately the changes in normal physiological variables, such as heart rate, blood pressure, sweating and body movement. Although variations in these parameters can be associated with the changes in the level of anaesthesia, many studies have demonstrated that they are not completely reliable [13-21]. In particular, the agent monitoring is of no value when total intravenous anaesthesia is used.

A growing body of evidence suggests that anaesthetics, particularly volatile anaesthetics, may produce long-lasting harmful effects. In particular, elderly people often suffer severe side effects from anaesthesia, such that past a certain age general anaesthesia will not be applied and surgery is often avoided. Monk et al. [18] studied 1,064 patients in a prospective observational study of one-year postoperative mortality. They found that "deep hypnotic time" was an independent predictor of increased mortality. Some recommendations have been made to limit the dose of anaesthetics.

In summary, pain, awareness and discomfort can only be experienced in the brain, and therefore a device that can monitor and interpret the brain activity during anaesthesia will be critical for reducing the likelihood of these experiences during surgery. The study of consciousness and depth of anaesthesia monitoring is at the forefront of current international research which will address fundamental questions in medicine, biology, physics and engineering. It will result in new models, algorithms and indexes which will be specific and will be immediately useful in the development of new DoA devices that have the potential to greatly improve the comfort of patients, reduce the medical cost and avoid intraoperative awareness and all its consequences. 


\section{Current research}

Currently, there are three basic approaches to study consciousness: the philosophical, the physical, and the neuroscientific. The subject of consciousness has a number of unique features distinguishing it from other scientific subjects. Some have argued that the unique features of quantum physics can explain the mysteries of consciousness [12], but using physics to explain consciousness is at present theoretically controversial and experimentally unproven. Although the ultimate explanation may, indeed, require a novel application of physics, it is the neuroscientific approach that has established a successful starting point for the endeavour. However, consciousness is a complex experience whose defining properties are still hotly debated by neuroscientists [12-14]. It is not as easy to pinpoint a single anatomical source of unconsciousness during anaesthesia. One leading theory holds that it is simply the result of "cognitive binding". Cognitive binding is thought to occur within and across sensory modalities and is thought to be necessary, although not sufficient, for consciousness itself. There are several proposed mechanisms for cognitive binding in the brain, including binding by convergence, binding by assembly, and binding by synchrony. Binding by convergence is the transmission of information from more primary processing areas to another region of the brain for integration. Binding by assembly refers to information being synthesized or bound in a Hebbian cell assembly, i.e., a group of inter-related neurons whose connections grow stronger with repeated firing together. Binding by synchrony is the coordination of neural firing in time and is thought to be associated with neural events at the frequency of $40 \mathrm{~Hz}$. A discussion of the strengths and weaknesses of each of these proposed mechanisms can be found in [12].

Cognitive binding by convergence denotes the process of the lower-order neural processing becoming synthesized in higher-order brain regions. Such neuronal subpopulations have been found to be selectively responsive to specific sensory stimuli. Although binding by convergence suggests a neural information integration in space, binding by synchrony suggests a neural information integration in time. There is abundant evidence for synchronization at all levels of neural processing that has been associated with perceptual tasks and cognitive binding [12]. There has been considerable focus on cortical activity in the band frequency of $40 \mathrm{~Hz}$ as a mechanism of binding. It has been suggested that cortical $40-\mathrm{Hz}$ activity coordinated by the thalamus serves to bind information that is processed within the $12-15 \mathrm{~ms}$ timeframe in which these oscillations propagate across the brain [12]. The $40 \mathrm{~Hz}$ oscillations of thalamo-cortical circuits have been proposed as neural correlates of consciousness and will become particularly relevant in the consideration of general anaesthetic mechanism. In 2005,
John and Prichep [15] described the "anaesthetic cascade" theory. Their proposed mechanism of general anaesthesia endorsed the concept of cognitive unbinding, while at the same time postulating a specific stepwise process by which anaesthetics suppress consciousness.

The EEG is, however, one of the oldest measures of the brain activity and continues to be popular both in clinical practice and in research. It is a comparatively cheap, robust, and straightforward technique, but nevertheless provides data with millisecond time resolution showing clear correlation with observed mental states. Its main shortcoming is a lack of spatial resolution. However, many questions about the state of the brain do not require perfect, or even any, spatial information. The hypnotic state is a likely candidate, since the absence of pain experience and memory retention suggests that gross changes of brain function have occurred. Thus in the last decade or so several heuristic approaches based on electroencephalographic monitoring of depth of anaesthesia have been developed [13, 16, 17]. Here we encounter a problem inherent to any heuristic approach: since it is not based on a deeper understanding of underlying mechanisms, failures do not point to improvements in an obvious manner [16].

In terms of the depth of anaesthesia measurement using EEG, the observation information $X$ will be a short EEG epoch and/or physiological changes, long enough to contain the necessary information, and short enough to allow frequent updates. We define states, $a, b$ and $c$, to correspond to the three extremes of the signal observational window, i.e., the awake, switch point and isoelectric EEG. As far as anaesthetic drugs are concerned, this window is sufficient since it is usually not desired to titrate patients beyond the point where cortical activity is totally suppressed. We first assume that states, $a, b$ and $c$, correspond to well established operating modes from which observation data are available. In this project, this assumption indicates that $a$ corresponds to the awake state (e.g., when a person is able to perform a mental task), $b$ corresponds to switch point (edge of consciousness and unconsciousness) and $c$ corresponds to an isoelectric signal (complete absence of cortical activity). Once we clearly define the states of the awareness of consciousness, we will then establish three reference data sets that correspond to the observations of the system in the states, $a, b$ and $c$. To characterise the data sets, we will choose and calculate a feature $f$ from each epoch. The feature $f$ will be derived in the original signal domain or in a transformed signal domain. Each epoch of the reference data sets is associated with a feature function. We will study the observation $X(d)$ if the system operates in an intermediate state $d$. Using the corresponding feature, we will estimate how far the system has evolved from the state $a$ toward the state $b$ and c by comparing the value $f(d)$ to the references $f(a), f(b)$ and $f(c)$. 
As we can see in this approach, the most important part is the anaesthetic feature extraction from the EEG. Unfortunately, the anaesthetic feature is unknown and indistinguishable in the measured EEG.

Recently, the author and other researchers propose to develop a mass neuronal thalamus-cortex model to interpret the invisible changes in the brain and to combine the identified features from measured frontal EEG signals with the physiological changes extracted from the proposed model to measure the DoA. The innovations of the proposed method are multiple: (1) to develop a mass neuronal thalamus-cortex model which relates the microscopic cellular and sub-cellular activities to their macroscopic consequences-induced EEG activity, (2) to predict the EEG signals on the scalp using a volume conductor computation technique, (3) to compare and fit the extracted features from measured real EEG and that from the predicted ones. This method will enable researchers to conduct an objective oriented DoA measurement and minimize the guess work from the assessment using a theoretical model which is based on known neurophysiology. This method, which is different from existing methods that infer the state of brain using only signal processing and data mining techniques, will redirect the current consciousness and DoA research from a heuristic approach to an algorithmic one, which will allow researchers to accumulate knowledge and to improve the outcomes continuously.

\section{Conclusion}

Every year, tens of millions of patients are exposed to general anaesthetics, drugs that remove the most precious human attribute - consciousness. About 50 million patients receive general anaesthesia each year in the US, as do 2 million patients in Australia.

The avoidance of side effects, such as depression of breathing and cardiac function, reduction in blood pressure and lowering of body temperature, relies on an increasing but variable array of monitoring devices. The attending anaesthetist, who is responsible to provide optimal surgical conditions and to ensure patient safety and comfort, must constantly calibrate drug delivery to assess the patient's condition and to achieve the desired depth-of-anaesthesia. The difficulty in tackling consciousness and depth-of-anaesthesia is for anaesthetists to determine what constitutes 'adequate' anaesthesia for an individual patient. Therefore, the consciousness research and the accurate DoA measurement have an inestimable value to both patients and anaesthetists, who are expecting an safe and reversible loss of consciousness in the surgery.

\section{References}

1. John ER, Prichep LS (2005) The anesthetic cascade: a theory of how anesthesia suppresses consciousness. Anesthesiology 102(2):447-471

2. Rampil IJ (2000) Mechanisms and sites of action of general anaesthetics, memory and awareness in anaesthesia IV. In: Jordan C, Vaughan DJA, Newton DEF (eds) Proceedings of the fourth international symposium on memory and awareness in anaesthesia. Imperial College Press, London, pp 223-231

3. Campagna JA, Miller KW, Forman SA (2003) Mechanisms of actions of inhaled anesthetics. N Engl J Med 348:2110-2124

4. Myles PS (2007) Prevention of awareness during anaesthesia. Best Pract Res Clin Anaesthesiol 21:345-355

5. Heyse B, Van Ooteghem B, Wyler B, Struys MM, Herregods L, Vereecke H (2009) Comparison of contemporary EEG derived depth of anesthesia monitors with a 5 step validation process. Acta Anaesthesiol Belg 60:19-33

6. Musizza B, Ribaric S (2010) Monitoring the depth of anaesthesia. Sensors 10:10896-10935

7. Myles PS, Leslie K, McNeil J et al (2004) Bispectral index monitoring to prevent awareness during anaesthesia: the B-Aware randomised controlled trial. Lancet 363:1757-1763

8. Nguyen KT, Wen P, Yan L (2009) Theoretical basis for identification of different anaesthetic states based on routinely recorded EEG during operation. Comput Biol Med 39(1):40-45

9. Nguyen-Ky T, Peng W, Yan L (2010) Improving the accuracy of depth of anaesthesia using modified detrended fluctuation analysis method. Biomed Signal Process Control 5(1):59-65

10. Nguyen-Ky T, Peng W, Yan L (2010) An improved de-trended moving average method for accurately monitoring the depth of anaesthesia. IEEE Trans Biomed Eng 57(10):2369-2378

11. Nguyen-Ky T, Peng W, Yan L, Gray R (2011) Measuring and reflecting depth of anaesthesia using wavelet and power spectral density techniques. IEEE Trans Inf Technol Biomed 15(4):630-639

12. Mashour GA (2006) Integrating the science of consciousness and anesthesia. Anesth Analg 103(4):975-982

13. Bowdle TA (2006) Depth of anaesthesia monitoring. Anesthesiol Clin 24:793-822

14. Orser BA (2007) Lifting the fog around anaesthesia. Sci Am 296(6):54-61

15. John ER, Prichep LS (2005) The anesthetic cascade: a theory of how anesthesia suppresses consciousness. Anesthesiology 102: 447-471

16. Bojak I, Liley DT (2005) Modelling the effects of anaesthesia on the electroencephalogram. Phys Rev 7(4):1-22

17. American Society of Anesthesiologists Task force on Intraoperative Monitoring (2006) Practice advisory for intraoperative awareness and brain functioning monitoring: a report. Anesthesiology 104(8):47-64

18. Monk TG, Saini V, Weldon BC et al (2005) Anaesthetic management and one-year mortality after non cardiac surgery. Anesth Analg 100:4-10

19. Monk TG, Weldon BC, Garvan CW, Dede DE et al (2008) Predictors of cognitive dysfunction after major non cardiac surgery. Anaesthesiology 108(1):18-30

20. Lindholm ML, Träff S, Granath F et al (2009) Mortality within 2 years after surgery in relation to low intraoperative bispectral index values and pre existing malignant disease. Anesth Analg 108:508-512

21. Davidson AJ, Huang GH, Czarnecki C et al (2005) Awareness during anaesthesia in children: a prospective cohort study. Anesth Analg 100:653-661 